\title{
EXCESSIVE FUNCTIONS OF CONTINUOUS TIME MARKOV CHAINS
}

\author{
BY JOHN G. KEMENY AND J. LAURIE SNELL ${ }^{1}$
}

Communicated by M. Kac, October 3, 1966

We consider transient continuous time Markov chains $P(t)$, with $P_{i j}{ }^{\prime}(0)=q_{i} \pi_{i j}$ for $i \neq j$ and $-q_{i}$ for $i=j$. We assume $0<q_{i}<\infty$ for all $i$. Then $1 / q_{i}$ is the mean time the process remains in state $i$, and $\pi$ is the transition matrix of the imbedded jump process. We let $q$ be a diagonal matrix with diagonal entries $q_{i}$.

A nonnegative function $h$ is $P(t)$-excessive (invariant) if $h \geqq P(t) h$, $(h=P(t) h)$ for all $t$. It is $\pi$-superregular (regular) if $h \geqq \pi h(h=\pi h)$. Our main results characterize the excessive functions of the minimal process in terms of $q$ and $\pi$. These results can also be used to characterize excessive functions of certain nonminimal processes.

Let $P^{\min }(t)$ be the minimal substochastic Markov transition matrix with the given $q$ and $\pi$. This is transient if and only if $\pi$ is transient. Let $G^{\mathrm{min}}$ be the Green's function of $P^{\mathrm{min}}$. A nonnegative function $h$

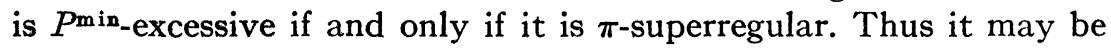
represented as $h=r+N f$, where $r$ is $\pi$-regular and $N f$ is a $\pi$-potential.

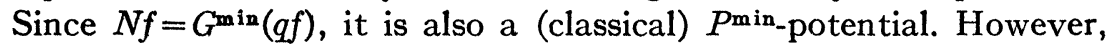

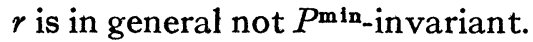

Let $B$ be the Martin boundary of $\pi$. Then $r=c \int_{B} K(\cdot, x) d \mu^{r}(x)$, where $\mu^{r}$ is harmonic measure for $\pi^{r}$, the " $h$-process" with $h=r$. Let $v_{i}$ be the probability that $P^{\min }$ started at $i$ stops in finite time, and $v^{h}$ be the corresponding vector for an $h$-process. We show that if $h$ is minimal, $\pi$-regular, then $v^{h}=0$ or 1 . This defines a partition: $B=B_{s}+B_{f}$ into slow $\left(v^{h}=0\right)$ and fast $\left(v^{h}=1\right)$ boundary points. This partition differs from the customary active-passive classification, in that the criterion is applied to $h$-processes. If we write $r=r_{s}+r_{f}$, integrating separately over $B_{s}$ and $B_{f}$, then $r_{s}$ is $P^{\text {min }}$-invariant and $r_{f}$ is a $P^{\text {min }_{-}}$ potential $\left(P^{\min }(t) r_{f} \rightarrow 0\right)$. Thus $r$ is a $P^{\text {min }}$-potential if and only if $\mu^{r}\left(B_{s}\right)$ $=0$. This potential is of class $D$ (Meyer) if and only if $\mu^{h}$ is absolutely continuous with respect to $\mu$. Thus we obtain many simple examples of nonclass $\mathrm{D}$ potentials.

These results are extended to a class of "instantaneous return processes" associated with a given $\pi$ and $q$. We obtain both criteria for a function to be excessive (in terms of $q$, $\pi$, and the return probabilities), and a representation of the excessive functions.

\section{Dartmouth College}

\footnotetext{
1 This research was supported by the National Science Foundation.
} 